\title{
Zur gravimetrischen Bestimmung des Interzellularraumvolumens von Pflanzenblättern
}

\author{
Von \\ H. Herrmann und L. Singer \\ Aus dem biologischen Institut der Carlsberg Stiftung, Kopenhagen \\ (Vorstand Dr. A. Fischer)
}

Die Grundlage der gravimetrischen Bestimmung des Interzellularraumvolumens (I.Z.R.V.) bildet die Infiltration des Interzellularraumes (I.Z.R.) mit Wasser. Die Differenz zwischen dem Gewicht des infiltrierten und des uninfiltrierten Blattes gibt unmittelbar die Größe des I.Z.R.V. an. Im folgenden soll kurz über Versuche berichtet werden, diese Infiltration durch Injektion des Wassers mit einer Injektionsspritze zu bewerkstelligen. Diese Art der Infiltration erlaubt eine Anwendung der gravimetrischen Bestimmung des I.Z.R.V. auch bei Blättern, die nicht von der Pflanze abgetrennt wurden. Die früher angewandten Infiltrationsmethoden (Zentrifugenmethode $^{1)}$ Infiltration des evakuierten I.Z.R. unter Druck ${ }^{2)}$ ) liessen diese Möglichkeit nicht zu. Unsere im folgenden beschriebene Injektionsmethode erfährt in ihrer Anwendbarkeit dadurch eine Einschränkung, daß sie nur bei homöobaren ${ }^{3)}$ und nicht zu dünnen Blättern durchgeführt werden kann.

Vor allem schien es uns wichtig einen Einblick in die Reproduzierbarkeit unserer Bestimmungen des I.Z.R.V. zu erhalten. Wir begannen daher diese Versuchsreihe mit Untersuchungen, die uns über diese Frage orientieren sollten. Dann erst versuchten wir die Bestimmung des I.Z.R.V. unabgetrennter Blätter. Schließlich bemühten wir uns, die Anwendbarkeit der von uns untersuchten Methode bei der Analyse des Wachstums von Pflanzenblättern durch die Bestimmung des I.Z.R.V. verschieden alter Blätter zu demonstrieren.

Methodik: Als Versuchsobjekt verwandeten wir Blätter von Gartenzyklamen. Zur Infiltration wird eine der Hauptblattadern der Blattunterseite, senkrecht zu ihrer Verlaufsrichtung durchstochen, so da $B$ die Injektionsnadel nach dem Durchstich unter der Blattepidermis der Blattunterseite fortgeführt werden kann. Das Gewebe der Blattader umgreift dabei eng anschließend die Injektionsnadel und verhindert so weitgehend bei der folgenden Injektion ein Ausfließen der Injektionsflüssigkeit aus dem Einstichkanal. Hat man die Nadel einige Millimeter weit knapp unter der Epidermis fortgeführt, so drückt man sanft auf den Spritzenstempel. Dabei sieht man die Injektionsflüssigkeit rasch in den I.Z.R. einflieBen. Dort, wo eine Infiltration stattgefunden hat, wird das Blatt in der Aufsicht dunkel in der Durchsicht hell. Bereits wenige Milligramm 
injizierten Wassers erzeugen eine scharf umschriebene, von der Umgebung stets eindeutig unterscheidbare Helligkeitsänderung, die sich bei Zyklamen besonders an der Blattunterseite mit freiem Auge ohne jede Schwierigkeit wahrnehmen läßt. Um den gesamten I.Z.R. zu füllen, ist es oft notwendig, zwei bis drei Einstiche an jedem Blatt vorzunehmen.

Bei der Untersuchung der Reproduzierbarkeit der gravimetrischen Bestimmung des I.Z.R.V. begnügten wir uns nicht mit der Prüfung der Injektionsmethode, sondern zogen zum Vergleich die Zentrifugenmethode heran.

Zur Kontrolle der Messungsergebnisse der Injektionsmethode stellten wir folgende Versuche an: Es wurde das Gewicht $\left(G_{1}\right)$ eines Blattes ermittelt. Dann wurde der I.Z.R. vollständig infiltriert. In diesem Falle ist die Blattunterfäche vollkommen dunkel und keine lufthaltige Stelle, die sich als heller Fleck scharf abgrenzt zu bemerken. Nach der Infiltration wird das Blatt rasch außen sorgfältig abgetrocknet und sofort gewogen $\left(G_{2}\right)$. Die Differenz $G_{2}-G_{1}$ gibt das Gewicht des injizierten Wassers und damit gleichzeitig das I.Z.R.V. an. Nun liessen wir das Blatt an der Luft liegen, bis es das injizierte Wasser abgegeben hatte und sein Anfangsgewicht wieder erreicht war. Nun wurde die Infiltration wiederholt und das nunmehr ermittelte Gewicht des Infiltrier-Blattes $\left(G_{3}\right)$ mit

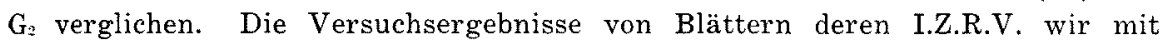
dieser Doppelbestimmung maßen sind in Tabelle 1 zusammengefaßt.

Tabelle 1

\begin{tabular}{c|c|c|r|r|r}
\hline $\begin{array}{c}\text { Blattgewicht } \\
\mathrm{G}_{1} \text { vor } \\
\text { Injektion }\end{array}$ & $\begin{array}{c}\text { Blattgewicht } \\
\mathrm{G}_{2} \text { nach erster } \\
\text { Injektion }\end{array}$ & $\begin{array}{c}\text { Blattgewicht } \\
\mathrm{G}_{3} \text { nach zweiter } \\
\text { Injektion }\end{array}$ & $\mathrm{G}_{2}-\mathrm{G}_{1}$ & $\mathrm{G}_{3}-\mathrm{G}_{1}$ & $\begin{array}{c}\text { Fehler } \\
\%\end{array}$ \\
\hline 719 & 957 & 945 & 238 & 226 & 5,2 \\
1148 & 1448 & 1442 & 280 & 294 & 4,8 \\
1658 & 2320 & 2316 & 662 & 658 & 0,5 \\
1839 & 2531 & 2552 & 692 & 713 & 3,2 \\
7799 & 1037 & 1055 & 238 & 256 & 7,1 \\
1054 & 1362 & 1384 & 308 & 330 & 6,7 \\
385 & 528 & 531 & 143 & 146 & 2,1 \\
1150 & 1465 & 1484 & 315 & 334 & 5,6 \\
917 & 1206 & 1187 & 289 & 270 & 7,0 \\
1111 & 1518 & 1511 & 407 & 400 & 1,8 \\
1179 & 1533 & 1527 & 354 & 348 & 1,7 \\
3446 & 4663 & 4628 & 1217 & 1182 & 1,8 \\
1397 & 1845 & 827 & 448 & 430 & 4,0 \\
880 & 1278 & 1259 & 398 & 379 & 4,7 \\
& & & & &
\end{tabular}

Die Gewichte sind in dieser Tabelle in $\mathrm{mg}$ angegeben. Da als Infiltrationsflüssigkeit Wasser verwendet wurde, kann man die Milligramm injizierten Wassers gleich setzen dem I.Z.R.V. in cbmm.

Der maximale Fehler bei diesen Doppelbestimmungen beträgt demnach $7,1 \%$.

Der Quotient $\frac{\text { I.Z.R.V. }}{\text { Blattgewicht }}$ beträgt im Durchschnitt 0,320, d.h. auf eine Einheit des Blattgewichtes entfallen 0,320 Teile I.Z.R.V

Den gleichen Versuch stellten wir auch mit der Zentrifugenmethode an. Die Infiltration der Blätter erfolgte in einer Zentrifuge bei einer Tourenzahl von $1800 \mathrm{Umdrehungen/Min.} \mathrm{In} \mathrm{Tabelle} 2$ sind die Versuchsresultate in gleicher Weise wie in Tab. 1 zusammengestellt. 
Tabelle 2

\begin{tabular}{c|c|c|c|c|c}
\hline $\begin{array}{c}\text { Blattgewicht } \\
\mathrm{G}_{1} \text { vor dem } \\
\text { Zentrifug. }\end{array}$ & $\begin{array}{c}\text { Blattgewicht } \\
\mathrm{G}_{2} \text { nach dem } \\
\text { 1. Zentrifug. }\end{array}$ & $\begin{array}{c}\text { Blattgewicht } \\
\mathrm{G}_{3} \text { nach dem } \\
\text { 2. Zentrifug. }\end{array}$ & $\mathrm{G}_{2}-\mathrm{G}_{1}$ & $\mathrm{G}_{3}-\mathrm{G}_{1}$ & $\begin{array}{c}\text { Fehler } \\
\%\end{array}$ \\
\hline 670 & 946 & 958 & 276 & 288 & 4,3 \\
243 & 545 & 531 & 302 & 288 & 4,8 \\
1160 & 1683 & 1702 & 523 & 542 & 3,5 \\
649 & 1005 & 1015 & 356 & 366 & 2,8 \\
756 & 1071 & 1086 & 215 & 230 & 6,5 \\
1448 & 2067 & 2066 & 619 & 618 & 0,2 \\
1766 & 2609 & 2614 & 843 & 848 & 0,5 \\
535 & 793 & 795 & 258 & 260 & 0,7 \\
469 & 715 & 735 & 246 & 266 & 7,5 \\
381 & 572 & 580 & 191 & 199 & 4,0
\end{tabular}

Bei dieser Methode beträgt der größte Fehler 7,5\%. Der Quotient $\frac{\text { I.Z.R.V. }}{\text { Blattgewicht }}$ beträgt im Durchschnitt 0,5. Der große Unterschied zwischen diesen Quotienten in den beiden Versuchen war auffallend. Bei einer Wiederholung des Versuches mit 12 Blättern, von denen 6 injiziert und 6 zentrifugiert wurden, konnten wir diesen Befund bestätigen. Der Quotient betrug bei den injizierten Blättern 0,412, bei den zentrifugierten Blättern 0,535.

Zur Entscheidung der Frage ob dieser Befund reell und methodisch bedingt ist, untersuchten wir 10 Blätter auf folgende Weise. Das zu untersuchende Blatt wurde gewogen $\left(G_{1}\right)$, in der Zentrifuge vollständig infiltriert und wieder gewogen $\left(\mathrm{G}_{*}\right)$. Dann wurde das Blatt bis zur Erreichung des Anfangsgewichtes getrocknet, diesmal mit der Injektionsspritze vollständig infiltriert und abermals gewogen $\left(G_{3}\right)$. Noch einmal auf das Anfangsgewicht gebracht, wieder zentrifugiert und ein letztes Mal gewogen $\left(G_{4}\right)$.

Dabei ergab sich, daß die Werte für das I.Z.R.V. die wir aus der doppelten Infiltration mit der Zentrifuge erhalten hatten, innerhalb der von uns gefundenen Grenze schwankten, während die Infiltration mit der Injektionsspritze zu Werten führte, die um etwa $15 \%$ tiefer lagen. Hinsichtlich ihrer Reproduzierbarkeit sind also die beiden Methoden gleichwertig. Die Injektionsmethode liefert aber die niedrigeren Absolutwerte. Diese Tatsache scheint auf Grund einer orientierenden mikroskopischen Untersuchung dadurch erklärbar, daß bei der Zentrifugenmethode die Infiltrationsflüssigkeit auch zwischen die dicht nebeneinanderstehenden Zellen des Palisadengewebes geprest wird, während diese bei der Injektion nur das eigentliche Schwammparenchym erfüllt.

Diesen Versuchen schlossen wir weitere an, um zu ermitteln ob die gravimetrische Bestimmung des I.Z.R.V auch an Blättern die nicht von der Pflanze abgetrennt sind durchführbar ist. Die Möglichkeit solcher Messungen schien uns ein wesentlicher Vorteil der Injektionsmethode. 
Mit einer Tuberkulinspritze, die mit einer Einteilung von $0,01 \mathrm{ccm}$ ausgestattet ist und bei der man $0,005 \mathrm{ccm}$ ohne weiteres schätzen kann, wurde das betreffende Blatt injiziert und das injizierte Wasservolumen an der Spritze abgelesen. Die aus dem Einstichkanal abfließende Flüssigkeit, die wir durch Auswahl geeigneter Einstichstellen möglichst einzuschränken suchten, wurde in einem gewogenen Wägegläschen aufgefangen und ev. an dem Blatte außen haftende Flüssigkeitströpfchen mit einem ebenfalls gewogenen Löschpapierstückchen abgetrocknet. Die Gewichtszunahme $G$ des Wägegläschens und Löschpapieres wurde bestimmt und von dem gesamten injizierten Flüssigkeitsvolumen $V$ abgezogen. Die Differenz V-G gibt die tatsächlich injizierte Wassermenge und damit gleichzeitig das I.Z.R.V. an. Zur Kontrolle bestimmten wir das I.Z.R.V. bei diesen Versuchsblättern durch direkte Wägung der Gewichtszunahme nach der Injektion, wie wir es oben beschrieben haben. Tabelle 3 gibt die Resultate der direkten und indirekten Wägung an.

Tabelle 3

\begin{tabular}{c|c|c}
\hline \multicolumn{2}{c|}{ I.Z.R.V. in cbmm } & Fehler in $\%$ \\
\hline direkte Wägung & indirekte Wägung & \\
\hline 100 & 106 & 6,0 \\
145 & 157 & 8,2 \\
73 & 67 & 8,2 \\
316 & 325 & 2,9 \\
1537 & 1620 & 5,3 \\
637 & 677 & 6,2 \\
2107 & 2203 & 4,5
\end{tabular}

Der Fehler beträgt also $8,2 \%$ und liegt mithin in Grenzen, die die Methode brauchbar erscheinen lassen. Innerhalb dieser Fehlergrenze ist die Injektionsmethode für die Bestimmung des I.Z.R.V von Blättern an der Pflanze anwendbar.

Anwendung der Methode. Während der Arbeit an dem rein methodischen Teil dieser Arbeit fiel uns auf, daß ganz kleine, junge, noch nicht einmal ganz entfaltete Blätter ein besonders kleines I.Z.R.V. im Verhältnis zu ihrem Gewicht zeigten. Wir mußten daran denken, ob nicht eine Abhängigkeit zwischen dem I.Z.R.V. und dem Blattgewicht besteht. Wie Tabelle 4 zeigt, konnten wir an drei Zyklamen die am Beginn der Vegetationsperiode untersucht wurden, eine Parallelität zwischen dem Blattgewicht und dem Quotienten I.Z.R.V. $\frac{\text { Blattgewicht }}{\text { beobachten. }}$

Tabelle 4

\begin{tabular}{|c|c|c|c|c|c|}
\hline \multicolumn{2}{|c|}{ Versuchspflanze 1} & \multicolumn{2}{|c|}{ Versuchspflanze 2} & \multicolumn{2}{|c|}{ Versuchspflanze 3} \\
\hline Blattgew. & $\frac{\text { I.Z.R.V. }}{\text { Blattgew. }}$ & Blattgew. & $\frac{\text { I.Z.R.V. }}{\text { Blattgew. }}$ & Blattgew. & $\frac{\text { I.Z.R.V. }}{\text { Blattgew. }}$ \\
\hline $\begin{array}{r}273 \\
935 \\
2137 \\
7435\end{array}$ & $\begin{array}{l}0,154 \\
0,331 \\
0,341 \\
0,495\end{array}$ & $\begin{array}{r}480 \\
1090 \\
2330 \\
2725 \\
4260 \\
8429\end{array}$ & $\begin{array}{l}0,376 \\
0,394 \\
0,458 \\
0,468 \\
0,497 \\
0,521\end{array}$ & $\begin{array}{r}432 \\
560 \\
968 \\
1068 \\
1275 \\
1692 \\
2354\end{array}$ & $\begin{array}{l}0,318 \\
0,335 \\
0,379 \\
0,414 \\
0,439 \\
0,445 \\
0,505\end{array}$ \\
\hline
\end{tabular}


Wir heben absichtlich hervor, daß es sich um Pflanzen handelte, die am Beginn der Vegetationsperiode der Zyklamen untersucht wurden. Denn es zeigte sich bald, daß ältere Pflanzen Unregelmäßigkeiten in dieser Gesetzmäßigkeit aufweisen und ganz alte Pflanzen diese vollkommen vermissen lassen. Dagegen bestätigte sich die oben erwähnte Beobachtung, daß die ganz kleinen Blättchen, mit dem für junge Blätter charakteristischen Glanz der Blattoberfläche immer wieder das relativ kleinste I.Z.R.V. aufwiesen. In der folgenden Tabelle 5 bringen wir drei weitere Reihen von Bestimmungen des I.Z.R.V. an Versuchspflanzen, die dieses Verhalten zeigen. Die Versuchspflanze 1 trug drei Blätter von dem erwähnten jugendlichen Typus, die beiden anderen Versuchspflanzen je eines (die jugendlichen Blätter sind immer die ersten in der Tabelle).

Tabelle 5

\begin{tabular}{|c|c|c|c|c|c|}
\hline \multicolumn{2}{|c|}{ Versuchspflanze 1} & \multicolumn{2}{|c|}{ Versuchspflanze 2} & \multicolumn{2}{|c|}{ Versuchspflanze 3} \\
\hline Blattgew. & $\begin{array}{c}\text { I.Z.R.V. } \\
\text { Blattgew. }\end{array}$ & Blattgew. & $\begin{array}{c}\text { I.Z.R.V. } \\
\text { Blattgew. }\end{array}$ & Blattgew. & $\frac{\text { I.Z.R.V. }}{\text { Blattgew. }}$ \\
\hline $\begin{array}{r}138 \\
203 \\
223 \\
270 \\
583 \\
640 \\
1596 \\
1790 \\
2599 \\
4380\end{array}$ & $\begin{array}{l}0,154 \\
0,182 \\
0,182 \\
0,315 \\
0,384 \\
0,421 \\
0,340 \\
0,480 \\
0,451 \\
0,450\end{array}$ & $\begin{array}{r}88 \\
270 \\
910 \\
1890 \\
3704\end{array}$ & $\begin{array}{l}0,193 \\
0,559 \\
0,486 \\
0,503 \\
0,440\end{array}$ & $\begin{array}{r}113 \\
198 \\
236 \\
491 \\
2470 \\
4370\end{array}$ & $\begin{array}{l}0,160 \\
0,262 \\
0,275 \\
0,429 \\
0,380 \\
0,436\end{array}$ \\
\hline
\end{tabular}

Überblicken wir also diese Versuche, so glauben wir folgendes zum Wachstum des Blattes bemerken zu dürfen: Durch die verschiedene Beteiligung der Komponenten am Wachstum eines Blattes kann dieses auf Grund der Bestimmungen des Blattgewichtes und des I.Z.R.V in zwei Phasen aufgelöst werden. In der ersten Phase sehen wir eine mächtige Entfaltung des I.Z.R., während die Gewichtszunahme relativ in den Hintergrund tritt. Solange sich die Blätter einer Pflanze in diesem Stadium befinden, sieht man eine weitgehende Parallelität zwischen dem Blattgewicht und dem Quotienten I.Z.R.V. Blattgewicht (Vgl. Tab. 4). In der zweiten Phase geht die Vergrößerung des I.Z.R.V etwa in gleichem Maße vor sich, wie die Zunahme des Blattgewichtes. In dieser Phase zeigt der Quotient I.Z.R.V. Blattgewicht keine Abhängigkeit vom Blattgewicht, sondern schwankt nur innerhalb enger Grenzen. Über den Rahmen dieser Arbeit hinaus, scheint die Möglichkeit zu bestehen, auch an Blätter, die sich an der Pflanze befinden, Bestimmungen des I.Z.R.V. mit Hilfe 
der Injektionsmethode zu machen. Diese Bestimmungen lassen eine genauere Analyse des Wachstums von Pflanzenblättern und der Beeinflußbarkeit einzelner Komponenten desselben zu.

Wir möchten uns noch erlauben, Herrn Prof. Dr. BoYsen Jensen für seine Kritik und Hilfe bei dieser Arbeit unseren verbindlichsten Dank auszusprechen.

\section{Zusammenfassung}

1) Es wird die Genauigkeit der gravimetrischen Bestimmung des Interzellularraumvolumens von Zyklamenblättern geprüft, wobei zur Infiltration des Interzellularraumes sowohl die Injektion als auch die Zentrifuge verwendet werden.

2) Die Fehlerbreite beider Methoden ist ziemlich gleich und beträgt etwa $7 \%$. Die Zentrifugenmethode gibt die höheren Absolutwerte.

3) An Blättern verschiedenen Alters wird die Beziehung des Qu otienten $\frac{\text { Interzellularraumvolumen }}{\text { Blattgewicht }}$ zum Blattgewicht untersucht.

\section{Literatur}

1) F. Weber, Vitale Blattinfiltration. Protoplasma, Bd. 1. 581, 1927.

2) P. Boysen-Jensen, Die Stoffpropuktion der Pflanze, S. 28. Jena 1932.

3) F. W. Neger, Spaltôffnungsschlu $\beta$ und künstliche Turgorsteigerung. Ber. d. deutsch. Bot. Ges. 30. 179, 1912. 